\title{
Efetividade da oxigenação por membrana extracorpórea (ECMO) no tratamento de pacientes com Covid-19: revisão sistemática
}

\author{
Effectiveness of extracorporeal membrane oxygenation (Ecmo) in the treatment of patients \\ with Covid-19: systematic review
}

\author{
Amanda Costa Araujo ${ }^{1}$ \\ Orcid: https://orcid.org/0000-0003-2740-8252 \\ Arthur Felipe Giambona Rente ${ }^{3}$ \\ Orcid: https://orcid.org/0000-0003-4238-4746
}

\author{
Délcio Uezato Junior ${ }^{2}$ \\ Orcid: https://orcid.org/0000-0002-7815-0901 \\ Dafne Port Nascimento 4 \\ Orcid: https://orcid.org/0000-0002-8474-727X
}

\begin{abstract}
Resumo
Introdução: O uso da Oxigenação por Membrana Extracorpórea (ECMO) vem sendo estudado no tratamento de pacientes com COVID-19, uma vez que essa terapia já demonstrou resultados favoráveis em outras patologias. Objetivos: Verificar a efetividade do uso da ECMO nos pacientes com insuficiência respiratória aguda grave causada pelo COVID-19. Material e Métodos: Para seleção dos artigos utilizaram-se os descritores controlados (DECS): ("Extracorporeal Membrane Oxygenation*" OR ECMO OR "Extracorporeal Life Support*" OR ECLS) AND ("COVID*" OR Coronavirus OR "SARS*"). As buscas foram realizadas nas bases de dados COCHRANE LIBRARY, PubMed, EMBASE e CINAHL. As variáveis analisadas foram os benefícios e a segurança desse tratamento, bem como se houve a redução da taxa de mortalidade, as contraindicações e os riscos com esse tratamento. Resultados: Após a consulta nas bases de dados, 292 artigos foram identificados utilizando os descritores mencionados acima, dos quais 11 foram incluídos nesse estudo após todo o processo de seleção. Os pacientes que necessitaram de ECMO eram predominantemente idosos que possuíam como fatores de risco: hipertensão arterial sistêmica, doença meningocócica e obesidade. As principais complicações encontradas foram fenômenos hemorrágicos e tromboembólicos. Discussão: Em relação às características laboratoriais, pode-se observar principalmente um aumento do nível de lactato, linfopenia e aumento da proteína $\mathrm{C}$ reativa. Os dados encontrados vão de encontro a outros estudos sobre o tema. Conclusão: Portanto, por se tratar de uma condição de saúde recentemente estudada, é necessário que novos estudos sejam conduzidos para confirmar a eficácia da ECMO em pacientes com COVID-19.
\end{abstract}

Palavras-chave: ECMO, oxigenação por membrana extracorpórea, covid-19.

\begin{abstract}
Introduction: The use of Extracorporeal Membrane Oxygenation (ECMO) has been studied in the treatment of patients with COVID-19, since this therapy has already shown favorable results in the treatment of acute respiratory failure of viral etiology, as in H1N1 influenza. Objectives: Analise the effectiveness of using ECMO in patients with severe acute respiratory failure caused by COVID-19. Material and Methods: For this purpose, a systematic literature review was carried out using the PICO structure (Patient, Intervention, Comparison and "Outcome") to formulate the guiding question. Controlled descriptors (DECS) were used to select the articles: ("Extracorporeal Membrane Oxygenation *" OR ECMO OR
\end{abstract}

\footnotetext{
${ }^{1}$ Programa de Mestrado Profissional em Inovação no Ensino Superior em Saúde, Universidade Municipal de São Caetano do Sul, Brasil. E-mail: amanda.araujo@online.uscs.edu.br

${ }^{2}$ Programa de Mestrado Profissional em Inovação no Ensino Superior em Saúde, Universidade Municipal de São Caetano do Sul, Brasil. E-mail: delcio.junior@online.uscs.edu.br

${ }^{3}$ Hospital Municipal de Emergências Albert Sabin, São Caetano do Sul, Brasil. E-mail: arthurfelipe3@gmail.com

${ }^{4}$ Programa de Mestrado e Doutorado em Fisioterapia, Universidade Cidade de São Paulo, Brasil. E-mail: dafnepn@yahoo.com.br
} 


\begin{abstract}
"Extracorporeal Life Support *" OR ECLS) and ("COVID *" OR Coronavirus OR "SARS *"). The searches were made in the COCHRANE LIBRARY, PubMed, EMBASE and CINAHL databases. Revisions, editorials, congress abstracts and study protocols were excluded. The variables analyzed were: The benefits and safety of this treatment, as well as whether there was a reduction in the mortality rate, contraindications and risks with this treatment. Results: After consulting the databases, 292 articles were identified using the descriptors mentioned above, of which 11 were included in this study after the selection process. The patients who needed ECMO were predominantly elderly and had the following risk factors: systemic arterial hypertension (SAH), meningococcal disease (DM) and obesity. The main complications found were hemorrhagic and thromboembolic. Finally, the mortality rate varied considerably between studies and it was possible to observe a significant decrease among patients who received this treatment. Discussion: The main results of this systematic review showed that the patients in this study were predominantly elderly and had underlying diseases mainly systemic arterial hypertension (SAH), meningococcal disease (DM) and obesity.

Conclusion: Therefore, as it is a recently studied health condition, it is necessary that further studies be conducted to confirm the effectiveness of ECMO in patients with COVID-19.

Keywords: ECMO, extracorporeal membrane oxygenation, covid-19
\end{abstract}

\section{Introdução}

No período de dezembro de 2019, a província de Wuhan, localizada na região central da China, registrou o primeiro caso de pneumonia viral associado à grave insuficiência respiratória ${ }^{1}$. $\mathrm{O}$ agente responsável, SARS-Cov-2, causador da doença nomeada como COVID-19 ${ }^{1}$. Desde então a doença se espalhou pelo mundo, sendo reconhecida como pandemia pela Organização Mundial da Saúde (OMS) em março de $2020^{1}$. Apesar de uma proporção significativa dos indivíduos serem assintomáticos ou apresentarem sintomas leves, aproximadamente 15 a $20 \%$ dos doentes podem desenvolver formas mais severas da doença, precisando de terapia intensiva e suporte ventilatório em consequência da síndrome da angústia respiratória aguda (SARA) ${ }^{2}$.

Hong $\mathrm{X}$ et al. recentemente constataram que dos 138 pacientes diagnosticados com COVID-19, 26,1\% necessitaram de cuidados intensivos, destes $61,1 \%$ evoluíram com SARA ${ }^{3}$. Atualmente, a taxa de mortalidade nos casos críticos pode chegar a $61,5 \%$, e nenhum tratamento específico foi comprovado até $\mathrm{o}$ momento $^{3,4}$. Nos casos de hipoxemia refratária, ou seja, nos casos graves de SARA, nos quais ocorre uma manutenção do quadro de falência respiratória a despeito da inundação e do colapso dos espaços aéreos impossibilitando a entrada de gás inspirado, o direcionamento dos casos se torna mais difícil, já que todas as possibilidades com evidência científica comprovadas são escassas ${ }^{5,6}$.

Dessa forma, terapias de suporte avançado com evidência científicas ainda não são totalmente estabelecidas no COVID-19, como as técnicas de suporte cardiorrespiratório que devem ter seus riscos e benefícios avaliados ${ }^{6}$. A oxigenação por membrana extracorpórea (ECMO) é uma forma de suporte cardiopulmonar por longo período em que o sangue é drenado do seu local inicial e devolvido à circulação depois de passar por um oxigenador por membrana e um transformador de calor ${ }^{2}$. No contexto da insuficiência respiratória aguda de origem infecciosa viral, existem alguns relatos prévios à COVID-19 sobre o uso da ECMO, principalmente referente à influenza $\mathrm{A}$ H1N1, no ano de $2009^{6}$.

Apesar de não existirem ensaios controlados aleatorizados com o tema COVID-19, foram publicadas algumas recomendações da OMS e da Extracorporeal Life Support Organization (ELSO). A ELSO é uma organização responsável pelos protocolos de uso, registro, informação e pesquisa em ECMO em nível mundial. De acordo com a OMS, a ECMO pode ser usada em pacientes com hipoxemia refratária apesar das medidas recomendadas, desde que seja realizada em 
centros especializados no manejo da $\mathrm{ECMO}^{3,7,8}$. Além disso, a ELSO indica a ECMO para pacientes com alta chance de desfecho fatal, principalmente nos quadros de SARA que evoluem com hipoxemia severa persistente, com valor de saturação de oxigênio no sangue ( $\mathrm{SpO} 2)$ menor que $80 \%$ por mais de 6 horas ou menor que $50 \%$ por 3 horas. Ou em pacientes com sinais de disfunção orgânica secundária ao menor aporte de oxigênio 9 .

Assim sendo, o objetivo primário desse estudo foi verificar a efetividade do uso da ECMO nos pacientes com insuficiência respiratória aguda grave causada pelo COVID-19. Os objetivos secundários foram apontar os desfechos do tratamento, efeitos colaterais, complicações e índice de diminuição da mortalidade.

\section{Materiais e Métodos}

\section{Amostra e tipo de estudo}

Foram utilizadas as bases de dados: Cochrane Library, PubMed (US National Library of Medicine Nation alI Institutes of Health), COCHRANE LIBRARY, EMBASE e CINAHL, utilizando os descritores controlados (DeCS- Descritor de Ciência da Saúde): ("Extracorporeal Oxygenation*" OR ECMO OR "Extracorporeal Life Support*" OR ECLS) AND ("COVID*" OR Coronavirus OR "SARS*"). Para a junção das palavras foi utilizado o operador booleano "AND". A data utilizada para as buscas foi do inicio das bases até 13/07/2020, quando a busca foi realizada.

A busca com os termos-chave nas bases de dados foi realizada por um dos autores. A coleta inicial dos artigos filtrados foi realizada em dupla (AA e DJ) a fim de concluir o processo de seleção e critérios de elegibilidade dos mesmos. No caso de discordâncias um terceiro avaliador (DN) foi consultado a fim de entrar em um consenso.

\section{Delineamento da pesquisa}

Foi elaborada uma revisão sistemática utilizando a estratégia PICO (Paciente, Intervenção, Comparador e "Outcome" (Desfecho)) para formulação da pergunta clínica desse estudo, sendo ela:

Pacientes: Pacientes com insuficiência respiratória aguda grave causada pelo COVID-19;

com o uso de ECMO;

Intervenção: Tratamento

- Comparador: Pacientes graves que foram tratados com o auxílio da ECMO, em comparação aos que não receberam esse recurso.

- Desfecho: Efetividade do tratamento com a ECMO.

Com isso formulou-se a pergunta: Qual efetividade na utilização da ECMO durante o manejo clínico dos pacientes com insuficiência respiratória aguda grave causada pelo COVID-19?

\section{Critérios de Inclusão e Exclusão}

Foram incluídos todos os artigos selecionados nas bases de dados acima descritas que respeitaram os seguintes critérios de inclusão: 1- Textos publicados na integralidade; 2- Que tenham como objetivo avaliar a efetividade do tratamento dos pacientes com COVID-19 utilizando a ECMO; 3- Estudos clínicos realizados com indivíduos diagnosticados com COVID-19. Não houve restrição por língua de publicação dos artigos incluídos.

Foram excluídos dessa revisão sistemática artigos que eram revisões, editoriais, resumos de congresso e protocolos de estudos, assim como os artigos que não preencheram os critérios de inclusão acima descritos.

\section{Procedimentos}

Inicialmente, a triagem foi realizada por título e resumo. Os artigos selecionados dessa triagem foram lidos com o texto completo do artigo. A extração dos dados foi realizada a partir da leitura completa dos estudos, sendo elas:

- $\quad$ Efetividade do tratamento;

- Desfechos do tratamento; 
complicações;

Efeitos colaterais e

- Índice de diminuição da mortalidade.

\section{Análise dos dados}

Não foi possível avaliar a qualidade metodológica dos estudos incluídos por se tratarem de desenhos heterogêneos. Além disso, os resultados foram apresentados de forma descritiva devido à diversidade de dados obtidos. Os dados coletados foram em número, porcentagem, média e desvio padrão.

\section{Resultados}

Foram identificados duzentos e quarenta e três artigos utilizando os descritores citados anteriormente. No entanto, findado todo o processo de seleção descrito na seção materiais e métodos desse estudo restaram apenas onze estudos. A Figura 1 ilustra o processo de seleção dos artigos:

Figura 1. Fluxograma da seleção dos artigos.

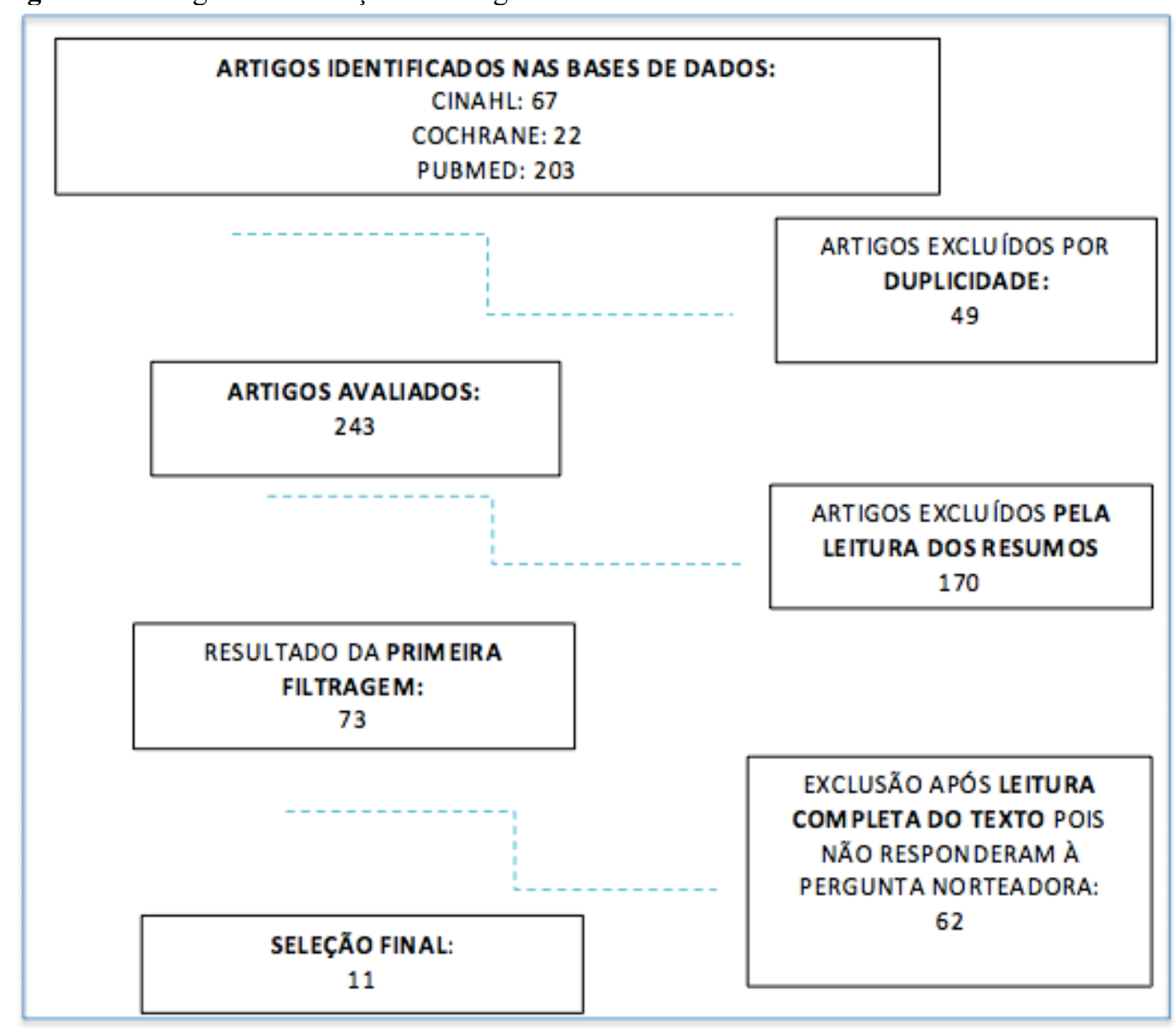

A Tabela 1 resume a quantidade de artigos encontrados em cada base de dados consultada, em números absolutos e em porcentagem. Sendo a maioria dos artigos da base de dados PubMed.

Tabela 1. Números absolutos e porcentagens de artigos encontrados nas bases de dados $(n=281)$ consultadas neste estudo.

Base de dados

\section{Número total de estudos $(n=292)$}

Número de artigos após a aplicação
dos critérios de inclusão e exclusão $(n=11)$ 


\begin{tabular}{lcccc} 
& $\mathrm{N}$ & $\%$ & $\mathrm{~N}$ & $\%$ \\
\hline CINAHL & 67 & 22,94 & 0 & 00,0 \\
\hline Cochrane & 22 & 7,53 & 0 & 00,0 \\
\hline PubMed & 203 & 69,52 & 11 & $100,0 \%$ \\
\hline
\end{tabular}

Todos os artigos foram publicados no idioma inglês, sendo que $54,4 \%$ foram desenvolvidos no Continente Europeu. Quanto ao ano de publicação, 100\% dos artigos foram publicados em 2020 em diferentes periódicos, como descritos na Tabela 2.

Tabela 2. Autor, ano, país, periódico e idioma dos artigos incluídos neste estudo.

\begin{tabular}{|c|c|c|c|}
\hline Autor & Ano de publicação & Periódico & País \\
\hline Beyls B et al. ${ }^{10}$ & 2020 & British Journal of Anaesthesia & França \\
\hline Falcoz PE et al. ${ }^{11}$ & 2020 & $\begin{array}{l}\text { American Journal of Riratory } \\
\text { and Critical Care Medicine }\end{array}$ & França \\
\hline Haye $\mathrm{G}$ et $a l .{ }^{12}$ & 2020 & $\begin{array}{c}\text { Journal of Cardiothoracic and } \\
\text { Vascular Aneshesia }\end{array}$ & França \\
\hline Huette $\mathrm{P}$ et al. ${ }^{13}$ & 2020 & Canadian Journal of Anesthesia & França \\
\hline Liu J et al. ${ }^{14}$ & 2020 & Medicine & China \\
\hline Loforte A et al. ${ }^{15}$ & 2020 & Asaio Journal & Itália \\
\hline Marullo A et al. ${ }^{16}$ & 2020 & Minerva Cardioangiologica & Itália \\
\hline Osho AA et al. ${ }^{17}$ & 2020 & Annals of Surgery & EUA \\
\hline Sultan I et al. ${ }^{18}$ & 2020 & Journal of Cardiac Surgery & EUA \\
\hline Yang X et al. ${ }^{19}$ & 2020 & Critical Care Medicine & China \\
\hline Xin L et al. ${ }^{20}$ & 2020 & Asaio Journal & China \\
\hline
\end{tabular}

Os artigos apresentaram como objetivo comum a comparação dos desfechos clínicos dos pacientes submetidos à ECMO devido à falência respiratória causada pelo SARS-CoV-2. A amostra totalizou 439 pacientes, tendo uma variação de 6 a 333 pacientes por estudo, a idade média dos pacientes foi de 47,13 (DP: 7,2) anos de idade.

Os sintomas respiratórios foram relatados em todos os estudos, sendo a hipoxemia grave o achado mais frequente. Quanto às comorbidades prévias, as mais encontradas foram a hipertensão arterial sistêmica e diabetes mellitus ${ }^{11,12,14,15,17,18,20}$. A Tabela 3 resume 
as principais características clínicas e epidemiológicas dos pacientes.

Tabela 3. Principais características clínicas e epidemiológicas dos pacientes.

\begin{tabular}{|c|c|c|c|c|}
\hline Autores & Amostra & Idade média & Sintomas & Comorbidades prévias \\
\hline Beyls C et al. ${ }^{10}$ & 12 & 62 & Hipoxemia grave & $\mathrm{N} / \mathrm{R}$ \\
\hline $\begin{array}{c}\text { Falcoz PE et } \\
a 1^{11}\end{array}$ & 17 & 56 & $\mathrm{~N} / \mathrm{R}$ & Obesidade, HAS, DM \\
\hline Haye G et al. ${ }^{12}$ & 8 & 57 & $\mathrm{~N} / \mathrm{R}$ & $\begin{array}{l}\text { Tabagismo, HAS, DM e } \\
\text { sobrepeso/obesidade }\end{array}$ \\
\hline $\begin{array}{l}\text { Huette P et } \\
a l^{13}{ }^{13}\end{array}$ & 14 & $\mathrm{~N} / \mathrm{R}$ & Hipoxemia grave & Doença renal \\
\hline Liu J et al. ${ }^{14}$ & 6 & 66 & $\begin{array}{l}\text { Febre, tosse, diarreia, } \\
\text { Mialgia }\end{array}$ & $\begin{array}{l}\text { HAS, Dm, Doença valvar, } \\
\text { transplante de fígado }\end{array}$ \\
\hline $\begin{array}{l}\text { Loforte A et } \\
\text { al. }{ }^{15}\end{array}$ & 4 & 49 & $\begin{array}{l}\text { Insuficiência } \\
\text { respiratória grave }\end{array}$ & Obesidade, HAS, DM \\
\hline $\begin{array}{l}\text { Marulo A } \\
\text { et al. }{ }^{16}\end{array}$ & 333 & 52 & $\mathrm{~N} / \mathrm{R}$ & $\mathrm{N} / \mathrm{R}$ \\
\hline $\begin{array}{c}\text { Osho AA et } \\
a \mathrm{l}^{17}\end{array}$ & 6 & 47 & $\mathrm{~N} / \mathrm{R}$ & DM e obesidade \\
\hline Sultan et al. ${ }^{18}$ & 10 & $\mathrm{~N} / \mathrm{R}$ & $\begin{array}{l}\text { Febre alta, tosse, } \\
\text { dispneia, } \\
\text { gastrointestinais }\end{array}$ & $\begin{array}{l}\text { HAS, DM, hiperlipidemia, } \\
\text { alcoolismo, tabagismo, } \\
\text { asma, apneia obstrutiva do } \\
\text { sono }\end{array}$ \\
\hline $\begin{array}{l}\text { Yang X et } \\
\quad \text { al. }{ }^{19}\end{array}$ & 21 & 65,5 & $\mathrm{~N} / \mathrm{R}$ & $\mathrm{N} / \mathrm{R}$ \\
\hline Xin L et al. ${ }^{20}$ & 8 & 64 & $\begin{array}{l}\text { Hipoxemia grave } \\
\text { refratária à } \\
\text { ventilação mecânica } \\
\text { invasiva }\end{array}$ & $\begin{array}{l}\text { HAS, DM, Hiperlipidemia, } \\
\text { alcoolismo, asma }\end{array}$ \\
\hline
\end{tabular}

Siglas: hipertensão arterial sistêmica (HAS) e doença meningocócica (DM).

*N/R: Não relatou.

Poucos estudos analisaram as características laboratoriais dos pacientes antes e durante a ECMO. Entre aqueles que relataram esses dados foi possível observar um predomínio de linfopenia e um aumento do lactato pré ECMO e linfocitose durante a $\mathrm{ECMO}^{10,13}$. A Tabela 4 resume todas as características laboratoriais relatadas pelos autores.

Tabela 4. Características laboratoriais pré e durante a ECMO. 


\begin{tabular}{|c|c|c|}
\hline Autores & $\begin{array}{c}\text { Características laboratoriais principais pré } \\
\text { ECMO }\end{array}$ & $\begin{array}{c}\text { Características laboratoriais } \\
\text { durante a ECMO }\end{array}$ \\
\hline Beyls C et al. ${ }^{10}$ & Aumento do lactato, linfopenia, plaquetopenia & Plaquetopenia \\
\hline $\begin{array}{c}\text { Falcoz PE et } \\
a l^{11}\end{array}$ & $\mathrm{~N} / \mathrm{R}$ & $\mathrm{N} / \mathrm{R}$ \\
\hline Haye G et al. ${ }^{12}$ & Linfopenia, proteína $\mathrm{C}$ reativa elevada & $\mathrm{N} / \mathrm{R}$ \\
\hline Huette $\mathrm{P}$ et al. ${ }^{13}$ & Linfopenia & $\begin{array}{l}\text { Linfocitose e degradação de } \\
\text { fibrinogênio }\end{array}$ \\
\hline Liu et al. ${ }^{14}$ & $\begin{array}{c}\text { Aumento do lactato, da creatinina, da bilirrubina } \\
\text { total, da proteína } \mathrm{C} \text { reativa e da procalcitonina e } \\
\text { plaquetopenia }\end{array}$ & $\mathrm{N} / \mathrm{R}$ \\
\hline Loforte A et al. ${ }^{15}$ & Linfopenia e neutropenia & $\mathrm{N} / \mathrm{R}$ \\
\hline Marulo A et al. ${ }^{16}$ & $\mathrm{~N} / \mathrm{R}$ & $\mathrm{N} / \mathrm{R}$ \\
\hline Osho AA et $a l^{17}$. & Aumento do lactato, D-dimero e da creatinina & $\mathrm{N} / \mathrm{R}$ \\
\hline Sultan I et al. ${ }^{18}$ & Elevação da ferritina e interleucina & $\mathrm{N} / \mathrm{R}$ \\
\hline Yang X et al. ${ }^{19}$ & $\mathrm{~N} / \mathrm{R}$ & $\begin{array}{l}\text { Leucocitose, linfocitose, } \\
\text { monocitose e aumento do d-dimero } \\
\text { e da creatinina }\end{array}$ \\
\hline Xin L et al. ${ }^{20}$ & Aumento do lactato & $\mathrm{N} / \mathrm{R}$ \\
\hline
\end{tabular}

Com relação à posição dos pacientes antes da colocação em ECMO, todos os pacientes foram posicionados na posição prona. Além disso, a maioria dos pacientes receberam o óxido nítrico $\mathrm{e} o$ anticoagulante em alguma fase do tratamento. Ao analisar os parâmetros respiratórios pré e pós ECMO observamos que na grande maioria dos estudos os parâmetros ventilatórios melhoraram após a ECMO, como mostra detalhadamente a Tabela 5 . 
Tabela 5. Parâmetros ventilatórios pré e pós ECMO

\begin{tabular}{|c|c|c|c|}
\hline Autores & $\begin{array}{c}\text { Parâmetros VM pré } \\
\text { ECMO }\end{array}$ & $\begin{array}{c}\text { Parâmetros VM } \\
\text { durante a ECMO }\end{array}$ & $\begin{array}{c}\text { Parâmetros VM pós } \\
\text { ECMO }\end{array}$ \\
\hline Beyls C et al. ${ }^{10}$ & $\begin{array}{l}\text { Volume corrente: } \\
6.1 \text {; } \\
\text { Frequência: } 30 \text { incursões por } \\
\text { minuto; PEEP: } 14 ; \\
\text { Pressão de platô: } 28 \text {; Pressão } \\
\text { de respiração: } 14 ; \\
\text { Complacência: } 31\end{array}$ & $\mathrm{~N} / \mathrm{R}$ & $\mathrm{N} / \mathrm{R}$ \\
\hline Falcoz PE et al. ${ }^{11}$ & $\begin{array}{l}\text { FIO2 (\%): 100; } \\
\text { Relação PaO2/FIO2, } \\
\text { (mmHg): 71; } \\
\text { SaO2 (\%): } 90 ; \\
\text { Volume total (ml/kg de peso } \\
\text { corporal previsto): } 5,9 ; \\
\text { Frequência respiratória: } \\
\text { (respirações/min): } 31 ; \\
\text { PEEP (cm de água): } 14 ; \\
\text { Pressão de platô cm de } \\
\text { água): } 29 ; \\
\text { Pressão de condução (cm de } \\
\text { água): } 15 ; \\
\text { Conformidade (ml/ cm de } \\
\text { água): } 26 ; \text { Índice de } \\
\text { oxigenação (cm de } \\
\text { água/mmHg): } 29\end{array}$ & $\begin{array}{l}\mathrm{SaO} 2: 97 ; \text { Volume total: } \\
3,9 ; \quad \text { Frequência } \\
\text { respiratória: 20; PEEP: } \\
\text { 12; Pressão de platô: } 26 ; \\
\text { Pressão de condução: } 14\end{array}$ & $\begin{array}{l}\text { Relação } \mathrm{PaO} 2 \text { /FIO2: } 177 \text {; } \\
\text { PEEP: 10; Pressão de platô: } \\
\text { 26; Pressão de condução: } 15 \text {; } \\
\text { Conformidade: } 29,5 \text {; Índice } \\
\text { de oxigenação: } 10\end{array}$ \\
\hline Haye $\mathrm{G}$ et al. $^{12}$ & $\begin{array}{l}\text { Volume corrente }(\mathrm{ml} \mathrm{kg} 1) \text { : } \\
\text { entre 4,2-6,5 } \\
\text { Frequência }(\mathrm{bpm}) \text { : entre } \\
30-35 \\
\text { PEEP (cm H2O): entre } 10 \text { e } \\
\text { 16; Pressão de respiração } \\
\text { (cm H20): entre } 9 \text { e } 20 \\
\text { Complacência }(\mathrm{ml} \mathrm{cm} \mathrm{H2O} \\
\text { 1): } 18-33 ; \mathrm{PaO} 2 / \mathrm{FiO} 2=\text { entre } \\
51 \text { e 95 }\end{array}$ & $\mathrm{N} / \mathrm{R}$ & $\mathrm{N} / \mathrm{R}$ \\
\hline Huette $\mathrm{P}$ et al. ${ }^{13}$ & $\begin{array}{l}\text { (PaO2/FIO2): } 76 \text { mmHg; } \\
\text { pressão de CO2: } 55 \mathrm{mmHg} \text {, } \\
\text { Complacência: } 30 \\
\text { mLmmHg. }\end{array}$ & $\begin{array}{l}\text { PaO2/FIO2: } 129 \text { mmHg; } \\
\text { Complacência: } \\
\text { mLmmHg }\end{array}$ & $\mathrm{N} / \mathrm{R}$ \\
\hline Liu J et al. ${ }^{14}$ & $\begin{array}{l}\text { PaO2/FiO2 96; PEEP: } 11.5 \text {; } \\
\text { volume: } 6.8 \text {; volume } \\
\text { corrente: } 525 \text {; Pressão na } \\
\text { inspiração: } 28.5 \text {; Frequência } \\
\text { respiratória: } 18\end{array}$ & $\mathrm{~N} / \mathrm{R}$ & $\begin{array}{l}\mathrm{PaO} 2 / \mathrm{FiO} 2329,4 ; \\
\text { 5.2; volume: } 9,3 ; \text { volume } \\
\text { corrente: } 650 ; \text { Pressão na } \\
\text { inspiração: } 20 ; \text { Frequência } \\
\text { respiratória: } 15\end{array}$ \\
\hline Loforte A et al. ${ }^{15}$ & $\begin{array}{l}\text { Ventilação (L/min): 11,5; } \\
\text { Volume corrente (ml): } \\
\text { 607,5); } \\
\text { PEEP (cm H2O); } \\
\text { FIO2 (\%) 95; } \\
\text { PaO2 /FIO2: 50,2; Pressão } \\
\text { de platô (cm H2O): } 31 ; \\
\text { Pressão de condução (cm } \\
\text { H2O): 16; Conformidade } \\
\text { (ml /cm H2O): 33,5 }\end{array}$ & $\mathrm{N} / \mathrm{R}$ & $\begin{array}{l}\text { Ventilação: de 4,1- } 5,1 \text {; } \\
\text { Volume corrente (ml): de } \\
\text { 280-320; } \\
\text { PEEP (cm H2O): de 10-12; } \\
\text { FIO2 (\%): de 50-60; Pressão } \\
\text { de platô (cm H2O): de 18-19; } \\
\text { Pressão motriz (cm H2O): de } \\
\text { 10-11; Conformidade: de } \\
\text { 33,2-34,1 }\end{array}$ \\
\hline
\end{tabular}




\begin{tabular}{|c|c|c|c|}
\hline Marulo A et al. ${ }^{16}$ & $\mathrm{~N} / \mathrm{R}$ & $\mathrm{N} / \mathrm{R}$ & $\mathrm{N} / \mathrm{R}$ \\
\hline Osho A et al. ${ }^{17}$ & $\begin{array}{l}\text { (média dos } 6 \text { pacientes): } \\
\text { Pressão de platô }=27,66 \mathrm{~cm} \\
\text { H2O; Volume }=5,23 \mathrm{~L} / \mathrm{min} \text {; } \\
\text { Fluxo de varredura do gás= } \\
93,33 \%\end{array}$ & $\mathrm{~N} / \mathrm{R}$ & $\begin{array}{l}\text { (média dos } 6 \text { pacientes): } \\
\text { Pressão de platô }=25,5 \mathrm{~cm} \\
\text { H2O; } \\
\text { Volume }=4,78 \mathrm{~L} / \mathrm{min} \text {; } \\
\text { Fluxo de varredura do gás= } \\
84,16 \%\end{array}$ \\
\hline Sultan I et al. ${ }^{18}$ & $\mathrm{~N} / \mathrm{R}$ & $\mathrm{N} / \mathrm{R}$ & $\mathrm{N} / \mathrm{R}$ \\
\hline
\end{tabular}

Não sobreviventes: Tinham uma necessidade contínua de

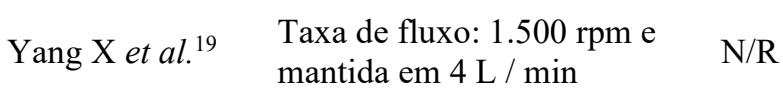
altas taxas de fluxo. Sobreviventes: O fluxo de varredura diminuiu gradualmente.

Xin L et al. ${ }^{20} \quad$ (PaCO2/FiO2): entre 54-76 N/R N/R

*N/R= não relatou.

Quanto aos desfechos e efeitos colaterais apresentados pelos pacientes observou-se que os dias de internação variaram de 4 a 60 dias, as principais complicações encontradas foram sangramentos, eventos trombóticos e alterações renais. Por fim, quanto à mortalidade observou-se uma considerável variação entre os estudos, com relato de $0 \%$ de mortalidade a $57,14 \%$ como mostra a Tabela 6.

Tabela 6. Desfechos apresentados pelos estudos analisados.

\begin{tabular}{|c|c|c|c|}
\hline Autores & $\begin{array}{l}\text { Média de dias } \\
\text { internados }\end{array}$ & $\begin{array}{c}\text { Desfechos e efeitos } \\
\text { colaterais }\end{array}$ & Complicações \\
\hline Beyls C et al. ${ }^{10}$ & 24 & $\begin{array}{l}\text { Alta da UTI: } 67 \% \text {; } \\
\text { Mortalidade: } 33 \%\end{array}$ & $\begin{array}{l}\text { Complicações trombóticas } \\
\text { durante a inserção da cânula; } \\
\text { tromboembolismo periférico } \\
\text { venoso; estado de } \\
\text { hipercoagubilidade em } \\
\text { pacientes com elevada } \\
\text { concentração de proteína C } \\
\text { reativa }\end{array}$ \\
\hline
\end{tabular}

Hemorragia; tamponamento cardíaco; AVC; TEP; trombose; pneumonia $\begin{array}{ll}\text { Falcoz PE } \text { et } \text { al. }{ }^{11} \quad \mathrm{~N} / \mathrm{R} & \begin{array}{l}\text { Alta hospitalar: } 58,5 \% \\ \text { Mortalidade: } 35,3 \%\end{array}\end{array}$ associada à ventilação mecânica; necessidade de terapia de substituição renal; embolia gasosa 


\begin{tabular}{|c|c|c|c|}
\hline Haye $\mathrm{G}$ et $a l^{12}$ & $\mathrm{~N} / \mathrm{R}$ & 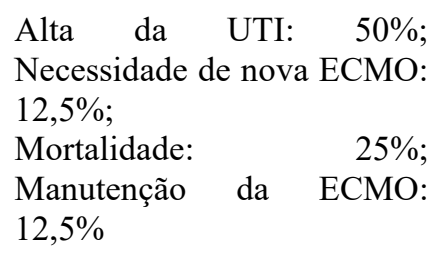 & $\begin{array}{l}\text { Sangramento; infecção da } \\
\text { cânula }\end{array}$ \\
\hline Huette $\mathrm{P}$ et al. ${ }^{13}$ & $\mathrm{~N} / \mathrm{R}$ & $\begin{array}{l}\text { Alta da UTI: } 67 \% ; \\
\text { Mortalidade: } 42,85 \%\end{array}$ & $\begin{array}{l}\text { Eventos trombóticos, } \\
\text { embolia pulmonar e } \\
\text { necessidade de terapia de } \\
\text { reposição renal }\end{array}$ \\
\hline Liu J et al. ${ }^{14}$ & 22 & $\begin{array}{l}\text { (após } 28 \text { dias) } \\
\text { Permaneceu em ventilação } \\
\text { mecânica: } 52 \% \text {; } \\
\text { Permaneceu na UTI: } 56 \% \text {; } \\
\text { Transferência para } \\
\text { enfermaria: } 22 \% \text {; } \\
\text { Alta hospitalar: } 22 \%\end{array}$ & $\mathrm{~N} / \mathrm{R}$ \\
\hline Loforte A et al. ${ }^{15}$ & 4 & Morte ou sobrevivência & $\begin{array}{l}\text { Sangramento gastrointestinal } \\
\text { grave }\end{array}$ \\
\hline Marulo A et al. ${ }^{16}$ & $\mathrm{~N} / \mathrm{R}$ & $\begin{array}{l}\text { Maior risco de morte em } \\
\text { idosos }(p=0,002) \text {; Desmame } \\
\text { da ventilação mecânica: } \\
18,1 \% \text {; } \\
\text { Mortalidade: } 17,1 \%\end{array}$ & $\mathrm{~N} / \mathrm{R}$ \\
\hline Osho A et al. ${ }^{17}$ & 17 & $\begin{array}{l}\text { Sobreviveu à alta hospitalar 1: } \\
\text { 17\%; Sobreviveu à } \\
\text { decanulação: }\end{array}$ & $\begin{array}{l}\text { Lesão renal } \\
\text { sangramento; }\end{array}$ \\
\hline Sultan I et al. ${ }^{18}$ & 18 & $\begin{array}{l}\text { Cura: } 20 \% \text {; } \\
\text { Permaneceu internado até a } \\
\text { finalização da pesquisa: } 10 \% \text {; } \\
\text { Mortalidade: } 10 \%\end{array}$ & Injúria renal aguda \\
\hline Yang X et al. ${ }^{19}$ & $\mathrm{~N} / \mathrm{R}$ & $\begin{array}{l}\text { Redução da mortalidade (sem } \\
\text { ECMO } 63,2 \% \text { versus com } \\
\text { ECMO } 57,1 \% ; p=0,782 \text { ) }\end{array}$ & $\begin{array}{l}\text { Bradicardia; sangramento no } \\
\text { local; hemorragia cerebral; } \\
\text { lesão renal aguda }\end{array}$ \\
\hline Xin L et al. ${ }^{20}$ & 60 & $\begin{array}{l}\text { Mortalidade: } 50 \% \text {; } \\
\text { terapêutico: } 37,5 \% \text {; } \\
\text { Permaneceu em } \\
12,5 \%\end{array}$ & $\mathrm{~N} / \mathrm{R}$ \\
\hline
\end{tabular}

N/R: Não relatou. 


\section{Discussão}

Os principais resultados dessa revisão sistemática mostraram que a amostra dos estudos era predominantemente idosa e possuía como doenças de base principalmente hipertensão arterial sistêmica (HAS), doença meningocócica (DM) e obesidade ${ }^{10-20}$. Quanto às características laboratoriais, pode-se observar principalmente um aumento do nível de lactato ${ }^{10,14,17,20 \text {, }}$ linfopenia ${ }^{10,12,13,15}$ e aumento da proteína $\mathrm{C}$ reativa $^{12,14}$. Pesquisadores ${ }^{21,22}$ evidenciaram que os pacientes com COVID-19 que evoluíram com a forma grave da doença possuíam os mesmos fatores de risco encontrados nesse estudo e também as mesmas alterações laboratoriais, como descrito por Rajagopal e colaboradores ${ }^{23}$.

Quanto aos efeitos colaterais, vale lembrar que a definição efeito colateral é toda reação prejudicial ou indesejada que ocorre durante ou após uma intervenção ou o uso de um medicamento ${ }^{22,24}$, sendo assim os possíveis efeitos colaterais associados à utilização da ECMO foram alterações sanguíneas como plaquetopenia ${ }^{10} \mathrm{e}$ degradação de fibrinogênio ${ }^{13}$ e bradicardia $^{19}$. Já, se tratando das complicações, definidas como evoluções desfavoráveis a despeito de um tratamento instituído ${ }^{24}$, dos onze estudos analisados, nove relataram algum tipo de complicação. Entre elas destacaram-se sangramentos, tanto no local da punção, quanto em diversos órgãos como cérebro e sistema gastrointestinal ${ }^{10,11,15,17,19}$, eventos tromboembólicos ${ }^{10,12}$, injúria renal ${ }^{11,13,17,18,19}$ e infecção no local da punção $^{12}$, além da pneumonia associada à ventilação mecânica ${ }^{11,13}$. Indo ao encontro, uma revisão realizada por Savarimuthu et al. $2020^{23}$, também relatou que as principais complicações encontradas nos pacientes em ECMO foram hemorragias, tanto no local da punção quanto em outros órgãos, como no cérebro $^{23}$, além de eventos tromboembólicos, infecções e pneumonia associada à ventilação mecânica ${ }^{23}$.
Por outro lado, a mortalidade variou consideravelmente de um estudo para o outro, sendo a menor taxa de mortalidade igual a $0 \%{ }^{14}$ e a maior igual a $57,14 \%{ }^{19}$, tal variação pode ser explicada pelo tamanho da amostra tão variável nos estudos, pela complexidade dos pacientes e do manejo clínico de uma doença com evolução incerta e pouco conhecida, assim como pela experiência do serviço com a utilização da ECMO. Dos estudos analisados apenas um estudo relatou diminuição da mortalidade de $63,2 \%$ para $57,1 \%(\mathrm{p}=0,782)^{19}$. Haiduc et al. ${ }^{26}$ também observaram uma redução da taxa de mortalidade nos pacientes que utilizaram a ECMO.

Além disso, observou-se que a ECMO vem sendo utilizada como terapia de resgate para os pacientes com insuficiência respiratória aguda causada pelo SARS$\mathrm{CoV}-2^{21}$, com isso, inúmeros pesquisadores estão descrevendo a sua experiência com essa estratégia terapêutica, no entanto, foi possível observar por meio dessa revisão sistemática que a maioria das publicações tratava-se de artigos não testados clinicamente como comentários, carta ao editor, editoriais, entre outros. Dessa forma, há um diminuto número de estudos que acompanhou e/ou comparou os pacientes que utilizaram essa terapia daqueles que não receberam o tratamento com a ECMO. Dessa forma, sugere-se que novos estudos sejam realizados a fim de confirmar os resultados obtidos nesse estudo.

\section{Conclusão}

Conclui-se que os pacientes que necessitaram de ECMO eram predominantemente idosos que possuíam como fatores de risco hipertensão arterial sistêmica (HAS), doença meningocócica (DM) e obesidade. As principais complicações encontradas foram fenômenos hemorrágicos e tromboembólicos. Por fim, a taxa de mortalidade variou consideravelmente entre os estudos sendo possível observar uma 
possível diminuição da taxa de mortalidade entre os pacientes que foram submetidos a esse tratamento. No entanto, por se tratar de uma condição de saúde recentemente estudada, é necessário que novos estudos sejam conduzidos para confirmar a eficácia da ECMO em pacientes com COVID-19.

\section{Referências Bibliográficas}

1. Associação de Medicina Intensiva Brasileira (AMIB). Preparação de URI para COVID19: lições de experiências internacionais. São Paulo [acesso em 30 mai. 2020]. Disponível em: https://www.amib.org.br/noticia/nid/preparacao-de-uti-para-covid-19licoes-de-experiencias-internacionais/

2. McIntosh K. Coronavirus disease 2019 (COVID-19). EUA [acesso em 01 jun. 2020]. Disponível em: https://www.uptodate.com/contents/coronavirus-disease-2019-covid19.

3. Hong X, Xiong J, Feng Z, Shi Y. Extracorporeal membrane oxygenation (ECMO): does it have a role in the treatment of severe COVID-19? Int J Infect Dis. 2020 May;94:7880. doi: 10.1016/j.ijid.2020.03.058.

4. Zeng Y, Cai Z, Xianyu Y, Yang BX, Song T, Yan Q. Prognosis when using extracorporeal membrane oxygenation (ECMO) for critically ill COVID-19 patients in China: a retrospective case series. Crit Care. 2020 Apr 15;24(1):148. doi: 10.1186/s13054-020-2840-8.

5. Patel BK. Insuficiência respiratória hipoxêmica aguda (IRHA, SARA). EUA [acesso em 10 jun. 2020]. Disponivel em: https://www.msdmanuals.com/pt/profissional/medicina-de-cuidadoscr $\% \mathrm{C} 3 \%$ ADticos/insufici $\% \mathrm{C} 3 \% \mathrm{AAncia}$-respirat $\% \mathrm{C} 3 \% \mathrm{~B} 3$ ria-eventila $\% \mathrm{C} 3 \% \mathrm{~A} 7 \% \mathrm{C} 3 \% \mathrm{~A} 30-\mathrm{mec} \% \mathrm{C} 3 \% \mathrm{~A} 2$ nica/insufici $\% \mathrm{C} 3 \% \mathrm{AAncia}-$ respirat $\% \mathrm{C} 3 \% \mathrm{~B} 3$ ria-hipox $\% \mathrm{C} 3 \% \mathrm{AAmica}-$ aguda-irha,-sara.

6. Ramanathan K, Antognini D, Combes A, Paden M, Zakhary B, Ogino M, et al. Planning and provision of ECMO services for severe ARDS during the COVID-19 pandemic and other outbreaks of emerging infectious diseases. Lancet Respir Med. 2020. doi:10.1016/S2213-2600(20)30121-1.

7. Extracorporeal Life Support Organization. ECMO para pacientes com COVID-19 com insuficiência cardíaca e/ou pulmonar. EUA [Acesso em 02 Jun 2020]. Disponível em: https://www.elso.org/Portals/0/Files/Guideline/ELSO_COVID19\%20Guidance\%20Document.Portugeuse\%20(1).pdf.

8. World Health Organization. Clinical management of severe acute respiratory infection when COVID-19 is suspected 2020. Suíça. [acesso em 30 maio.2020]. Disponível em: https:/www.who.int/publications-detail/clinical-management-of-severe-acuterespiratory-infection-when-novel-coronavirus-(ncov)-infection-is-suspected.

9. Barros L, Rivetti L, Furlanetto B, Teixeira E, Welikow A. COVID-19: general guidelines for cardiovascular surgeons (standard guidelines- subject to change). Brazilian Journal of Cardiovascular Surgery 2020. doi:10.21470/1678-9741-1-20200604 
10. Beyls C, Huette P, Abou-Arab O, Berna P, Mahjoub Y. Extracorporeal membrane oxygenation for COVID-19-associated severe acute respiratory distress syndrome and risk of thrombosis. Br J Anaesth. 2020;125(2):e260-e262. doi:10.1016/j.bja.2020.04.079.

11. Falcoz PE, Monnier A, Puyraveau M, Perrier S, Ludes PO, Olland A et al. Extracorporeal Membrane Oxygenation for Critically Ill Patients with COVID-19related Acute Respiratory Distress Syndrome: Worth the Effort? Am J Respir Crit Care Med. 2020 Aug 1;202(3):460-463. doi: 10.1164/rccm.202004-1370LE.

12. Haye G, Fourdrain A, Abou-Arab O, Berna P, Mahjoub Y. COVID-19 Outbreak in France: Setup and Activities of a Mobile Extracorporeal Membrane Oxygenation Team During the First 3 Weeks [published online ahead of print, 2020 May 8]. J Cardiothorac Vasc Anesth. 2020;S1053-0770(20)30424-9. doi:10.1053/j.jvca.2020.05.004

13. Huette P, Beyls C, Guilbart M, Coquet A, Berna P, Haye G, Roger PA et al. Extracorporeal membrane oxygenation for respiratory failure in COVID-19 patients: outcome and time-course of clinical and biological parameters. Can J Anaesth. 2020 Oct;67(10):1486-1488. doi: 10.1007/s12630-020-01727-z.

14. Liu J, Dong YQ, Yin J, He G, Wu X, Li J, Qiu Y, He X. Critically ill patients with COVID-19 with ECMO and artificial liver plasma exchange: A retrospective study. Medicine (Baltimore). 2020 Jun 26;99(26):e21012. doi: 10.1097/MD.0000000000021012.

15. Loforte A, Dal Checco E, Gliozzi G, Benedetto M, Cavalli GG, Mariani C et al. Venovenous Extracorporeal Membrane Oxygenation Support in COVID-19 Respiratory Distress Syndrome: Initial Experience. ASAIO J. 2020 Jul;66(7):734-738. doi: 10.1097/MAT.0000000000001198.

16. Marullo AG, Cavarretta E, Biondi-Zoccai G, Mancone M, Peruzzi M, Piscioneri F et al. Extracorporeal membrane oxygenation for critically ill patients with coronavirusassociated disease 2019: an updated perspective of the European experience. Minerva Cardioangiol. 2020 Apr 27. doi: 10.23736/S0026-4725.20.05328-1.

17. Osho AA, Moonsamy P, Hibbert KA, Shelton KT, Trahanas JM, Attia RQ et al. Venovenous Extracorporeal Membrane Oxygenation for Respiratory Failure in COVID-19 Patients: Early Experience From a Major Academic Medical Center in North America. Ann Surg. 2020 Aug;272(2):e75-e78. doi: 10.1097/SLA.0000000000004084.

18. Sultan I, Habertheuer A, Usman AA, Kilic A, Gnall E, Friscia ME et al. The role of extracorporeal life support for patients with COVID-19: Preliminary results from a statewide experience. J Card Surg. 2020 Jul;35(7):1410-1413. doi: 10.1111/jocs.14583.

19. Yang X, Cai S, Luo Y, Zhu F, Hu M, Zhao Y et al. Extracorporeal Membrane Oxygenation for Coronavirus Disease 2019-Induced Acute Respiratory Distress Syndrome: A Multicenter Descriptive Study. Crit Care Med. 2020 Sep;48(9):12891295. doi: 10.1097/CCM.0000000000004447. 
20. Li X, Guo Z, Li B, Zhang X, Tian R, Wu W et al. Extracorporeal Membrane Oxygenation for Coronavirus Disease 2019 in Shanghai, China. ASAIO J. 2020 May;66(5):475-481. doi: 10.1097/MAT.0000000000001172.

21. Wang D, Hu B, Hu C, Zhu F, Liu X, Zhang J et al. Clinical Characteristics of 138 Hospitalized Patients With 2019 Novel Coronavirus-Infected Pneumonia in Wuhan, China. JAMA. 2020 Mar 17;323(11):1061-1069. doi: 10.1001/jama.2020.1585.

22. Kon ZN, Smith DE, Chang SH, Goldenberg RM, Angel LF, Carillo JA et al. Extracorporeal Membrane Oxygenation Support in Severe COVID-19. Ann Thorac Surg. 2020 Jul 17:S0003-4975(20)31152-8. doi: 10.1016/j.athoracsur.2020.07.002.

23. Rajagopal K, Keller SP, Akkanti B, Bime C, Loyalka P, Cheema FH et al. Advanced Pulmonary and Cardiac Support of COVID-19 Patients: Emerging Recommendations From ASAIO-a Living Working Document. Circ Heart Fail. 2020 May;13(5):e007175. doi: 10.1161/CIRCHEARTFAILURE.120.007175.

24. Marodin G, Goldim JR. Confusiones y ambigüedades en la clasificación de eventos adversos en investigación clínica. Rev. esc. enferm. USP [Internet]. 2009 Sep [cited 2020 Oct 18];43(3):690-696. doi: 10.1590/S0080-62342009000300027.

25. Savarimuthu S, BinSaeid J, Harky A. The role of ECMO in COVID-19: Can it provide rescue therapy in those who are critically ill? J Card Surg. 2020 Jun;35(6):1298-1301. doi: 10.1111 jocs.14635.

26. Haiduc AA, Alom S, Melamed N, Harky A. Role of extracorporeal membrane oxygenation in COVID-19: A systematic review. J Card Surg. 2020 Oct;35(10):26792687. doi: $10.1111 /$ jocs.14879.

\section{Como citar este artigo:}

Araujo AC, Uezato Junior D, Rente AFG, Nascimento DP. Efetividade da oxigenação por membrana extracorpórea (ECMO) no tratamento de pacientes com covid-19: revisão sistemática. Rev. Aten. Saúde. 2021; 19(70): 249-262. 
